\title{
Bronchiectasis: What We Don't Know Yet But Should
}

\author{
Katy L.M. Hester, MBBS, MRCP1, Melissa McDonnell, MBBS, MRCP1,2,3 and Anthony De Soyza, PhD,
} MBChB, BMSc, FRCP1,3,4

${ }^{1}$ Institute of Cellular Medicine, Newcastle University and Adult Bronchiectasis Service, Newcastle upon Tyne Hospitals, Newcastle upon Tyne, UK;

${ }^{2}$ National University of Ireland, Galway, Ireland; ${ }^{3}$ EMBARC European Bronchiectasis Registry; ${ }^{4}$ BronchUK, UK Bronchiectasis Partnership

\section{ABSTRACT}

Bronchiectasis is a chronic lung disease characterized by airway dilatation, mucus retention, and recurrent lower respiratory tract infections. Bronchiectasis has a significant morbidity and an appreciable mortality rate and is increasing in prevalence. Despite this, there are still significant knowledge gaps in our understanding of the epidemiology, pathophysiology, prognosis, and optimal treatments in bronchiectasis. This review highlights selected key knowledge gaps in bronchiectasis. Addressing these gaps in knowledge could improve the disease burden both for patients and healthcare systems. Coordinated research networks and large database cohorts of patients ready to participate in both observational and intervention studies will be needed to improve our treatment options. (BRN Rev. 2016;2:14-26)

Corresponding author: Anthony De Soyza, Anthony.de-soyza@ncl.ac.uk

Key words: Bronchiectasis. Infection. Macrolide. Pseudomonas aeruginosa. Treatment adherence. 


\section{INTRODUCTION}

Bronchiectasis is increasingly recognised as both a primary disease and as a complication of other common diseases such as asthma and chronic obstructive pulmonary disease (COPD). There is a paucity of data on the epidemiology, pathophysiology, and optimal treatment approaches in this patient population. Data does suggest, however, that the incidence of bronchiectasis is increasing, with a corresponding increase in mortality rates. Marked heterogeneity exists in the aetiologies of bronchiectasis and these may result in different therapeutic targets. Significant knowledge gaps remain and there is a continuing need to systematically coordinate our research endeavours to enable us to deliver a step-wise change in bronchiectasis management from empirical to evidence-based therapies. Networks are developing to capture large patient cohorts, allowing better understanding of the disease process and its management (e.g. www.bronchiectasis.eu and BronchUK, www.bronch.ac.uk). Multidisciplinary and cross-national efforts in epidemiology, microbiology, genetics, immunology, basic science, and epithelial biology in conjunction with the pharmaceutical industry will help to tackle these knowledge gaps and deliver effective new therapies to the clinic. Selected research areas that such collaborative efforts may undertake are discussed in this article.

\section{DEFINITION AND DIAGNOSIS OF BRONCHIECTASIS}

Bronchiectasis, also referred to as non-cystic fibrosis bronchiectasis, is characterised by irreversible airway dilatation with clinical features including cough, chronic sputum production, haemoptysis, dyspnoea, and chronic rhinosinusitis. Patients report recurrent hospital admissions, reduced quality of life, and fatigue. ${ }^{1-4}$. The "vicious cycle" hypothesis describes how a combination of host susceptibility and environmental insult leads to progressive airway damage and dilatation $^{5}$. Defects in host immune response can also contribute to chronic infection and inflammation ${ }^{6}$.

Whilst bronchiectasis is quite frequently seen in COPD and in some cases of chronic asthma, it is unclear if there is causality or co-association between these conditions. Recognised aetiologies include post-infection, immunodeficiency syndromes, inflammatory bowel disease, connective tissue diseases, primary ciliary dyskinesia, Young's syndrome, and inhalation of a foreign body. A significant proportion of cases, up to $50 \%$, will have idiopathic bronchiectasis ${ }^{7-10}$. Investigations to identify associated conditions may include genetic testing for cystic fibrosis, Aspergillus precipitins, immunological profile, and sputum microbiology 9 . Defining the aetiology leads to management changes for both children and adults with bronchiectasis ${ }^{7,10,11}$.

To confirm clinical suspicions, high-resolution computed tomography scanning (HRCT) is required ${ }^{12}$. Studies acknowledge that a clinical scenario highly suggestive of bronchiectasis may not correlate with a "positive" HRCT, which raises questions about the links between clinical symptoms and the pathophysiological processes involved ${ }^{7,8}$. It has also been shown in two case-series that approximately $15 \%$ of radiologically diagnosed 


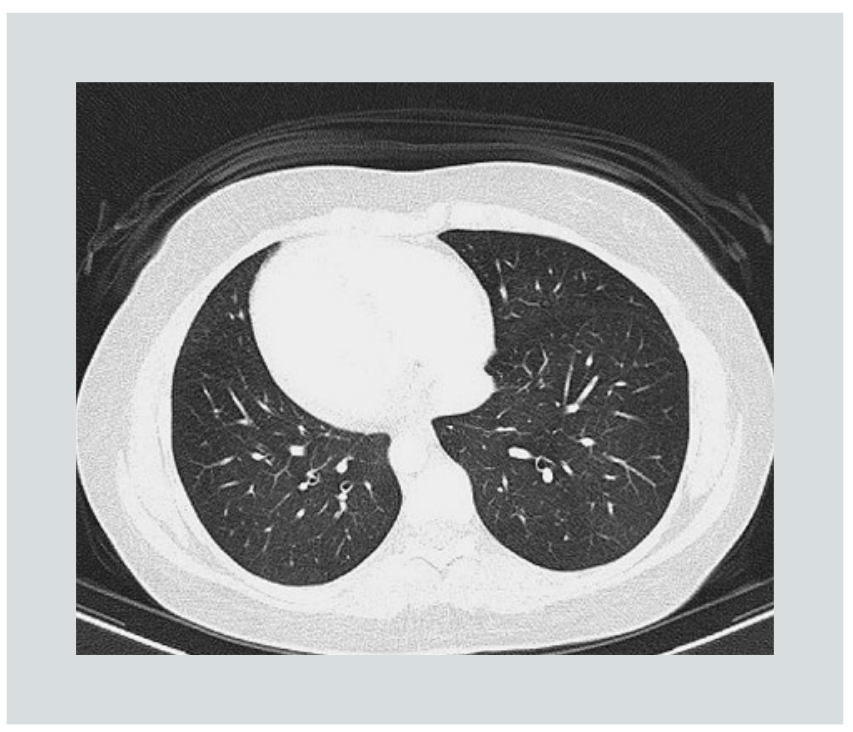

Figure 1. Does aetiology predict outcomes? This CT scan demonstrated Kartagener's syndrome with very mild fusiform bronchiectasis and dextrocardia. This patient has had four exacerbations per year, all manageable with oral antibiotics. Many clinicians report that patients with primary ciliary dyskinesia, including Kartagener's, run a milder course than other aetiologies. To date convincing multicentre data confirming this is rare. As more genetic understanding of primary ciliary dyskinesia develops, it remains to be understood if different genetic mutations leading to primary ciliary dyskinesia leads to different prognoses.

patients had their bronchiectasis diagnosis refuted on re-read of their scans ${ }^{8,13}$.

The comparison of airway and accompanying artery diameter in order to establish presence of airway dilatation has been described pathologically ${ }^{14}$. The same comparison is used to look for airway dilatation on HRCT scans ${ }^{15}$. Bronchial wall thickening is also well described in bronchiectasis imaging (Fig. 1 and 2). When bronchiectasis and COPD co-exist or overlap, understanding which process drives this is challenging. Gatheral et al. studied the impact of COPD-related bronchiectasis and showed the presence of bronchiectasis to be associated with increased infections and hospitalisations, regardless of bronchial wall

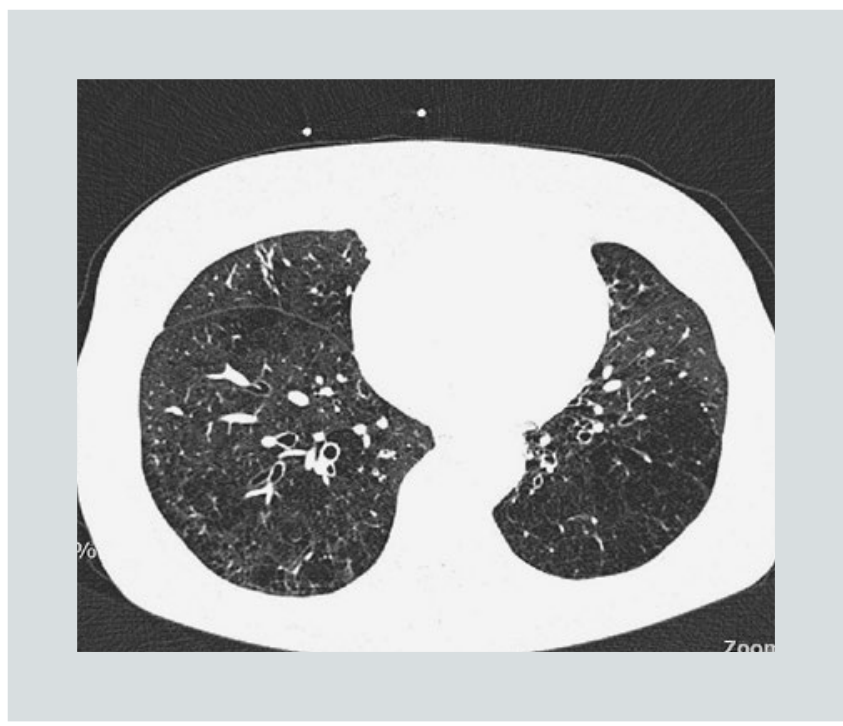

Figure 2. What are the minimal investigation sets required to define aetiology? This computed tomography scan demonstrates emphysema and bronchiectasis, suggesting the underlying aetiology may be bronchiectasis and COPD overlap syndrome. However, when referred to a specialist centre because of recurrent exacerbations, "basic tests" were undertaken. These and the clinical history revealed a childhood onset of symptoms and panhypogammaglobulinaemia with normal alpha-1 antitrypsin levels. This suggested a possible immunodeficiency associated bronchiectasis with co-existent smoking related COPD. Treatment with intravenous immunoglobulin markedly reduced infective exacerbation rates.

thickness ${ }^{16}$. Difficulty remains in defining COPD-driven bronchiectasis or idiopathic bronchiectasis in an ex- or current smoker. A catch-all term, bronchiectasis and COPD overlap syndrome (BCOS), has been suggested $^{17}$ (Table 1).

\section{EPIDEMIOLOGY}

Reported rates of bronchiectasis are likely to be inaccurate due to misdiagnosis (as other common respiratory disorders, e.g. asthma or COPD) and missed diagnosis (e.g. with overlapping diagnoses such as BCOS and asthma-bronchiectasis overlap syndrome [ABOS]). Data across multiple healthcare systems suggest that 
TABle 1. Known unknowns in definitions and diagnosis

1. What are the false positive and false negative rates of bronchiectasis on chest HRCT?

2. Can computerized algorithms help define bronchiectasis and monitor airway calibre changes on HRCT?

3. What is the natural history of suspected bronchiectasis patients with a "normal" HRCT? How many progress to bronchiectasis within five years?

4. What is the minimal set of aetiological investigations? (Fig. 2)

5. Does HRCT have any role in monitoring response to treatment? (Fig. 3)

6. Does air trapping/mosaicism pattern on HRCT predict a better response to anti-inflammatory treatments? (Fig. 4)

7. How good are HRCT patterns at directing investigations into aetiology such as ABPA or NTM disease?

8. What role is there for HRCT in helping define causes for recurrent exacerbations?

9. What clinical features suggest extended aetiological testing (e.g. cystic fibrosis genotyping, extended immunology testing) is undertaken?

10. What symptoms or clinical features in common respiratory diseases such as COPD and asthma should prompt HRCT scanning to exclude coexistent bronchiectasis?

There are several unknowns in the definitions and diagnosis of bronchiectasis. ABPA: allergic bronchopulmonary aspergillosis; NTM: non-tuberculous mycobacterial disease; HRCT: high-resolution computed tomography.

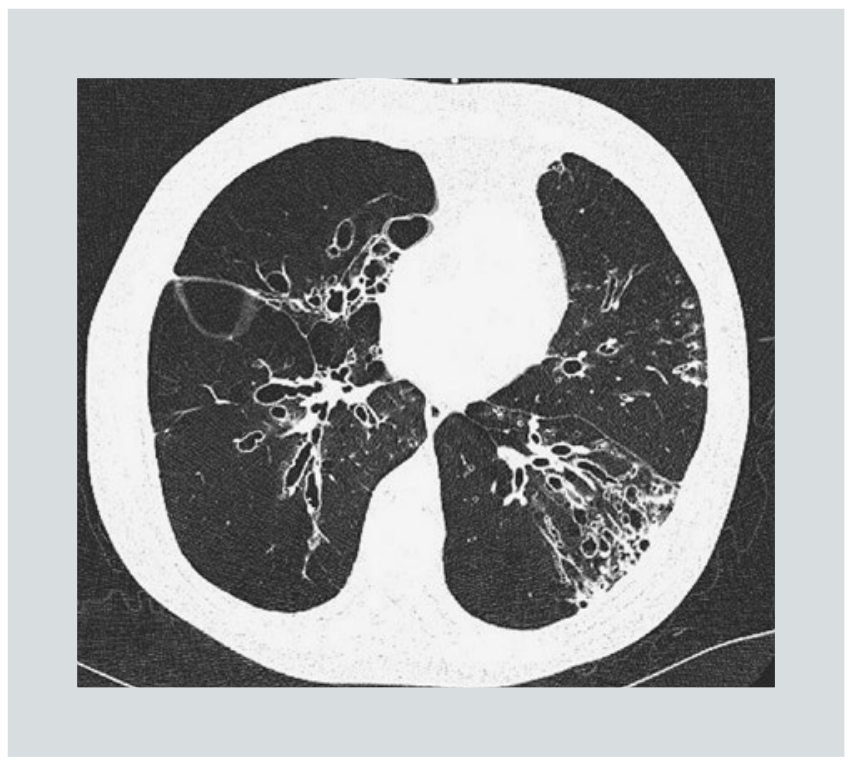

Figure 3. Do radiological patterns predict outcomes or help monitor response to treatment? This computed tomography demonstrates multilobar cystic bronchiectasis. Cystic bronchiectasis has been suggested as associated with a poorer prognosis. Disease extent on radiology such as the Reiff score is a component of the Bronchiectasis severity index (Chalmers et al. $)^{25}$. Many patients with such extensive cystic disease are infected with Pseudomonas aeruginosa. The role of computed tomography in assessing response to treatment or explaining why a patient gets recurrent exacerbations is unproven. The reasons why some patients develop cystic bronchiectasis and others have less florid disease is also unknown.

Establishing aetiological causality in bronchiectasis also presents on-going uncertainties: separating idiopathic bronchiectasis from post-infectious bronchiectasis is challenging. Accuracy in categorisation is important as some aetiological subgroups encountered in clinical practice are excluded from interventional studies ${ }^{22,23}$. A recent UK primary care database study suggested that HIV infection was a more common aetiological cause of bronchiectasis than previously reported ${ }^{21}$. This may reflect greater rates of HRCT scanning in this population, perhaps advocating wider use of HRCT in other "at-risk" groups. High rates of bronchiectasis in COPD have also been reported (as high as $40 \%$ ), yet this 


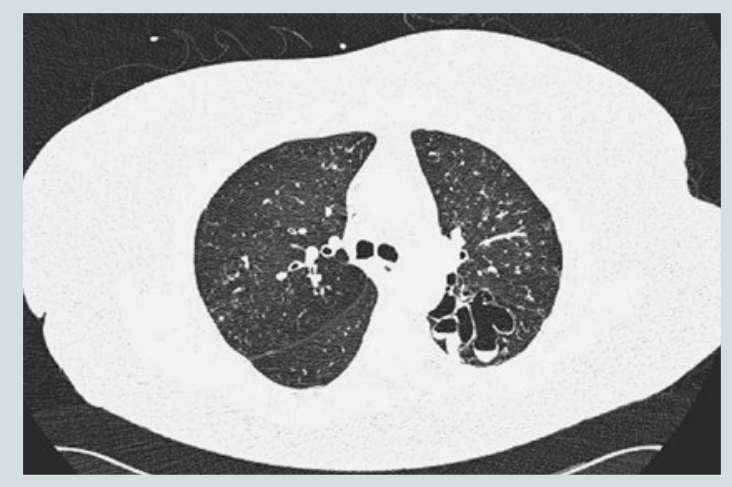

FigurE 4. Do computed tomography appearances help predict pathophysiology? This computed tomography demonstrates left lower lobe predominant cystic bronchiectasis with mild cylindrical bronchiectasis in other lobes. Careful review of the scan suggests mosaicism (areas of higher perfusion next to areas of lower perfusion), raising the possibility of a significant heterogeneity of small airways disease and gas trapping. Although this is relatively common in bronchiectasis, little is known about the mechanisms leading to this appearance in some but not all. A further unknown is whether this computed tomography appearance predicts better responses to inhaled corticosteroids or anti-inflammatory macrolides.

is likely to be influenced by which sub-population of COPD patients are investigated, i.e. "chronic bronchitis recurrent exacerbators" or all other phenotypes of COPD (such as emphysema without exacerbations) $)^{9,16}$. The fact that the conditions known to overlap with bronchiectasis (COPD and asthma) are so prevalent could translate into a large increase in identification of cases of bronchiectasis.

Several unknowns are listed in table 2.

\section{PROGNOSIS}

Until recently, prognosis data has been dominated by single-centre studies, often from specialised centres. Available data from across the
Table 2. Known unknowns in bronchiectasis epidemiology

1. Is there a genuine increase in prevalence or simply an improved diagnostic rate or change in diagnostic thresholds on HRCT?

2. Do aetiologies vary between developing and developed nations?

3. Which aetiologies are associated with a poorer outcome/early mortality?

4. Is there good inter-observer correlation when aetiological causes of bronchiectasis are attributed?

5. Can we develop more robust aetiological definitions that work in clinical practice?

6. Which aetiologies benefit from targeted specific treatment?

7. Is there an impact of national programs of pneumococcal vaccination on rates of bronchiectasis?

HRCT: high-resolution computed tomography.

UK suggests an increasing mortality rate at 3\% per year ${ }^{22}$. Two prognostic scoring indices providing estimates of mortality and hospitalisations over a 4-5 year period have recently been published: the FACED score (Forced expiratory volume 1 second, Age, Colonisation by Pseudomonas aeruginosa, Extent [number of lobes affected] and Dyspnoea, Spain) and the Bronchiectasis Severity Index (BSI, Edinburgh). The latter was internationally validated across four centres (UK, Belgium, and Italy). It has many components similar to the FACED with additional factors consisting of weighting for prior healthcare use and persistent infection with "other pathogens" ${ }^{24,25}$. Neither, however, has been tested outside of Europe or in developing nations and we do not know how interventions such as Pseudomonas eradication may alter such prognostic indices (Table 3).

\section{PATHOGENESIS AND MICROBIOLOGY}

Cole's continuous and self-perpetuating vicious cycle hypothesis provides a useful 
TABle 3. Known unknowns in bronchiectasis prognosis

1. Are there differences between the FACED and BSI prognostic scores in utility and accuracy?

2. What factors will influence uptake and use of prognostic indices into routine clinical practice?

3. Will clinicians alter management based on "high-risk" scoring in such systems and what treatment intensification might be applied in "high-risk" patients?

4. Do these scoring indices work effectively across different healthcare systems?

5. Do different aetiologies confer different prognoses? How does bronchiectasis and COPD overlap syndrome affect the performance of the scoring indices?

6. What is the impact of comorbidities on prognosis? Should we be assessing all patients with bronchiectasis for prevalent comorbidities?

7. Can these prognostic scores help transplant referral and predict mortality over a transplant window of 1-2 years?

8. Does the mortality rate or hospitalisation risk fall if Pseudomonas is "eradicated" and their FACED or BSI score falls?

FACED: forced expiratory volume 1 second, age, colonisation by Pseudomonas aeruginosa, extent (number of lobes affected) and dyspnoea score;

BSI: bronchiectasis severity index.

model of bronchiectasis pathophysiology to this day ${ }^{5}$. Treatment is targeted at interrupting the interconnecting processes of failed mucus clearance, airway bacterial colonisation, airway inflammation, and airway structural damage in an attempt to break this cycle and prevent disease progression ${ }^{26,27}$.

Airway inflammation is dominated by neutrophils, with impaired mucociliary clearance and failure of neutrophil opsonophagocytic killing ${ }^{6}$. Since these neutrophils are believed to be normal prior to their arrival in the airway, it is likely that the airway inflammatory milieu itself impairs bacterial clearance $^{27-29}$. Neutrophil elastase is associated with reduced opsonisation of pathogens, further promoting pro-inflammatory cytokine release. Neutrophil elastase also slows ciliary beat frequency and promotes mucus hypersecretion via activation of the MUC5AC gene $^{30,31}$. Further mechanisms of immune dysfunction include failure to clear apoptotic cells and T-cell infiltration, particularly Th17 cells $^{32}$. Much more work, however, is needed before targeted anti-cytokine therapies can be trialled.

Patients with bronchiectasis frequently develop acute infective pulmonary exacerbations characterised by symptoms of fever, purulent sputum, and dyspnoea, with associated adverse effects on both morbidity and quality of life. Frequent exacerbations may contribute to the progressive decline of lung function, although both the aetiology and pathophysiology of exacerbations remains poorly understood. Studies using classical microbial culture techniques in stable state bronchiectasis report Haemophilus influenzae (14-47\% of cases), Pseudomonas aeruginosa (5-31\%), and Streptococcus pneumoniae $(2-14 \%)$ as the most frequently isolated pathogens ${ }^{10,33,34}$.

Bronchiectatic airways may become "colonised" by $P$. aeruginosa with "repeated positive sputum cultures over a defined period of time". Conflicting data exist on the independent contribution of $P$. aeruginosa to long-term prognosis. Whether $P$. aeruginosa drives disease progression or is simply a marker of existing severe disease remains in question. A recent meta-analysis of 3,683 bronchiectasis patients demonstrated $P$. aeruginosa colonisation in $21.4 \%$ of patients, which was associated with a threefold increase in mortality and a sevenfold increase in hospitalisation rates ${ }^{35}$. In this analysis, eight different methods of defining $P$. aeruginosa colonisation in bronchiectasis studies were identified. The word 
"colonisation" is misleading and perhaps "persistent infection" should be adopted more widely.

Although there are numerous studies in cystic fibrosis $(\mathrm{CF})$ regarding the transmissibility of epidemic strains of $P$. aeruginosa and Mycobacterium abscessus, cross-infection data in bronchiectasis are very limited ${ }^{36-38}$. The 2010 guidelines failed to comment on this evidence gap, leaving it unclear as to whether or not bronchiectasis patients with $P$. aeruginosa should be segregated as is recommended in CF infection control guidelines ${ }^{9}$. Most cross-infection studies in bronchiectasis are single centre and focus on $P$. aeruginosa, with limited longitudinal sampling ${ }^{39-41}$. These often lack a robust description of the clinical environment, e.g. are facilities shared with patients who have $\mathrm{CF}$ and carry known $\mathrm{CF}$ epidemic strains? In a case series of 40 patients from a UK adult bronchiectasis clinic without strict segregation, using two genotyping techniques to cross validate findings, no single common "epidemic strain" was found ${ }^{39}$. However, certain common strains found widely within the natural environment, e.g. Pseudomonas clone $\mathrm{C}$, did infect patients raising the possibility of environmental acquisition in the bronchiectasis population ${ }^{39}$.

Pujana et al. examined 16 patients with $64 P$. aeruginosa isolates. Despite the limited discrimination potential of DNA fingerprinting, cross-infection, or common source, acquisition was absent ${ }^{40}$. In another study of 125 isolates taken from 31 patients attending a bronchiectasis clinic in Spain, pulsed field gel electrophoresis suggested that certain strains may be predominant within a clinical setting ${ }^{41}$.
On balance, there are no strong data suggesting widespread $P$. aeruginosa epidemic strains as a cause of clinical decline in bronchiectasis; why this is so different to CF remains unknown.

Whilst $P$. aeruginosa colonisation is associated with reduced lung function, a longitudinal study demonstrated $P$. aeruginosa infection across all stages of airflow limitation, highlighting the importance of rigorous sputum surveillance protocols in all bronchiectasis patients, even those with "mild" airflow limitation ${ }^{42}$.

Huge variations in definitions and therapies for bacterial eradication are noted between different healthcare systems and countries. Is it possible to fully eradicate $P$. aeruginosa? One retrospective study suggested that $34 \%$ of $P$. aeruginosa colonised patients subsequently became culture negative on follow-up; however, it is difficult to determine if this was true eradication or failure of successful in vitro culture using classical techniques ${ }^{42}$. The capability of $P$. aeruginosa to form biofilms protects it from both the immune system and systemically delivered antibiotics. It also rapidly adapts to chronic infection in the lung and readily develops antimicrobial resistance ${ }^{27}$.

The BTS guidelines for bronchiectasis recommend eradication treatment for new isolation of $P$. aeruginos $a^{8}$. However, some patients may spontaneously clear the organism without treatment. A recent randomised controlled trial evaluating the efficacy of three months nebulised tobramycin following intravenous antibiotics on initial isolation of $P$. aeruginosa, showed Pseudomonas-free rates of $54.5 \%$ in treatment group versus $29.4 \%$ in placebo group $^{43}$. These eradication rates are, however, significantly lower than those reported in CF, 
further confirming the different pathobiology of these two conditions.

We have few data on microbiology outside Europe and Australasia, and there is also a definite lack of data describing the impact of organisms other than P. aeruginosa in bronchiectasis, in particular in those colonised with the most common bronchiectasis pathogens such as $H$. influenzae ${ }^{35}$. Recent reports suggest that Haemophilus-infected patients do have a worse outcome compared to non-colonised patients, but to a lesser extent than P. aeruginosa $^{35}$. A recent post-hoc analysis of the trial suggested that in patients without $P$. aeruginosa airway infection, erythromycin did not significantly reduce exacerbations and actually promoted displacement of $H$. influenzae by more macrolide-tolerant pathogens including $P$. aeruginosa. These findings argue for a cautious approach to chronic macrolide use in patients without $P$. aeruginosa airway infection ${ }^{44,45}$.

Significant recent advances in our understanding of bronchiectasis have arisen through culture-independent microbiological techniques that allow a comprehensive analysis of polymicrobial bacterial communities in the lung ${ }^{27}$. Detection of uncultivable microorganisms has challenged our understanding pathogenesis, progression, and management of bronchiectasis. These technologies reveal colonisation with organisms previously not recognised by culture-based studies like Veilonella sp., Prevotella sp. and Neisseria spp ${ }^{50}$. Loss of microbiome diversity, with dominance of one or a few species, is associated with worse lung function and increased exacerbations $^{46,47}$. An inverse relationship between the abundance of $P$. aeruginosa and that of $H$. influenzae within the bronchiectasis lung
TABLE 4. Selected known unknowns in bronchiectasis microbiology

1. What is the best and most acceptable definition of bacterial "colonisation" or "eradication"?

2. Does $P$. aeruginosa always drive disease progression or is it a marker of disease severity?

3. What is the clinical relevance of a single isolate of $P$. aeruginosa and when should treatment be initiated?

4. What factors make $P$. aeruginosa more likely to occur (or persist) in some patients and not others?

5. Does chronic infection with $P$. aeruginosa always contribute to lung function decline?

6. Why does Pseudomonas appear to have lower resistance rates and lack of significant epidemic strain infections in bronchiectasis than seen in cystic fibrosis?

7. Can we successfully eradicate $P$. aeruginosa? What do we consider to be a successful eradication? Does this have any impact on disease outcomes?

8. Is there a pattern of polymicrobial colonisation that can be identified? Is there an inverse relationship between $H$. influenzae and $P$. aeruginosa and is this related to extent of lung damage?

9. Can the microbiome be manipulated to confer a better clinical outcome?

bacterial community suggests a progression in microbial states ${ }^{47}$.

The role of viruses and air pollution, whilst described in other disease areas, is poorly studied in bronchiectasis. Exacerbations in bronchiectasis are frequently managed with antibiotics; however, viral infections may also be significant in many cases. Recent data suggests that respiratory viruses may play a crucial role in triggering bronchiectasis exacerbations $^{48}$ (Table 4).

\section{TREATMENT}

There are a number of widely used current therapies in bronchiectasis; to our knowledge none are specifically licensed for the treatment 
of bronchiectasis. We will limit discussion to antimicrobials, though note there are many unknowns in the utility and best regimens for anti-inflammatory treatments, physiotherapy, and mucolytics in bronchiectasis. Many therapies developed and now used for $\mathrm{CF}$ do not readily translate across into bronchiectasis, e.g. nebulised aztreonam, now licensed for $\mathrm{CF}$, has two "negative" phase III trials in bronchiectasis $^{49}$. This was also seen for the inhaled mucolytic mannitol ${ }^{50}$. It is entirely plausible that good therapies for bronchiectasis have been inadvertently but inappropriately discarded due to unintended sub-optimal trial designs translated over from the $\mathrm{CF}$ literature.

Perhaps the greatest evidence of efficacy for treatments currently used in bronchiectasis is for prolonged or long-term macrolide (LTM) antibiotics with three large scale studies consistently showing an effect in exacerbation reduction with either long-term azithromycin $500 \mathrm{mg}$ thrice weekly, $250 \mathrm{mg}$ daily, or $400 \mathrm{mg}$ twice daily of erythromycin (BLESS, BAT and EMBRACE trials) ${ }^{51-53}$. Several recent meta-analyses, including these studies and various smaller scale studies, have confirmed the role for LTMs in exacerbation prevention $^{54-57}$. There were no clear effects on pathogen clearance or eradication, but statistical if not clinically significant improvements in forced expiratory volume in one second $\left(\mathrm{FEV}_{1}\right)$ and quality of life measures were observed. Unfortunately, a consistent theme, where measured, was the statistically significant increase in macrolide resistance. The evidence for macrolides in preventing exacerbations in recurrent exacerbators is strong. The role of LTM in patients with high symptom burdens but without frequent exacerbations is unclear. In our experience, many of these report
TABLE 5. Known unknowns with long-term macrolides

1. Do LTMs have an acceptable long-term safety profile in an elderly comorbid population?

2. Does macrolide-associated increased antimicrobial resistance in the upper airway flora translate into a later increase in the rate of resistance in the general population or of refractory pneumonia?

3. What are best markers of LTM response and how soon after treatment should patients be monitored/treatment stopped?

4. Can LTM responders be identified by any patient characteristic or biomarker in advance or is a therapeutic trial needed?

5. Can we withdraw macrolides after "stabilizing" a patient, and if so, what period of time does this take?

6. Should chronic macrolide therapy be restricted to patients with frequent exacerbations and high severity index scores or is there a rationale for using them in highly symptomatic but non-exacerbating "lower risk" patients?

7. How do we define partial response to macrolides? Do patients not responding to one macrolide respond to switching to an alternative macrolide or dose escalation?

8. Can LTM treatment holidays be applied to patients across a range of disease severities in spring/summer months?

9. Do the differing macrolides offer similar efficacy but differing adverse event profiles, e.g. ototoxicity, cardiac conduction defects and/or resistance induction rates?

Whilst there are a number of good quality studies of long-term macrolides in bronchiectasis, there are still several unanswered questions.

LTM: long-term macrolide.

symptomatic improvement with LTM. However, many questions remain over the use of long-term macrolides (Table 5).

Chronic inhalational antibiotics (CIA) are widely used in bronchiectasis and until recently the evidence base was scant. A single-centre single-blind study of gentamicin produced marked reductions in exacerbations; multicenter double-blind, follow-on studies may help truly define the utility of this therapy in daily practice $^{58}$. Data from a large multicenter international trial of nebulised ultrafine colistin delivered by an intelligent breath-activated nebuliser was particularly notable, even though the study failed to reach its primary endpoint. This landmark study 
demonstrated a significant proportion of trials participants were non-compliant. When a per protocol analysis was conducted, limiting the analysis to patients defined as compliant at $80 \%$ or more of prescribed therapy, there was a significant exacerbation reduction effect. These observations, along with others, suggest we have issues in defining the appropriate target population, the optimal outcome measures, and in ensuring that non-compliance does not impair study outcomes ${ }^{59}$.

Phase II studies have suggested a possible role for dry powder inhaled ciprofloxacin and nebulised liposomal ciprofloxacin, with a reduction in bacterial load ${ }^{60,61}$. Both are now in phase III development programs.

One key challenge will be to understand the implications of "resistant" strains in CIA therapy. Firstly, the breakpoints established for antimicrobials that are currently used to define resistance or sensitivity probably have little relevance to the biofilm mode of growth, nor the very high concentrations possible within the lung with CIA. Secondly, longterm CIA therapy will likely select a sub-population of resistant bacteria. If, however, the suppression of sensitive strains results in a clinically meaningful reduction in total bacterial load, the emergence (or selection) of resistant strains may be acceptable.

There are significant uncertainties in the role of either inhaled or oral long-term antibiotics. There are no studies randomising patients to LTM as compared to CIA. One uncertainty for the future will be where such new therapies fit in in comparison to long-term macrolides? Commonly, there is a patient preference for LTM, given these are easier to fit into a treatment regimen.
TABLE 6. Known unknowns in chronic inhaled antimicrobial therapy

1. Is CIA therapy an "add-on" to LTM or a first choice before LTM? Do patient characteristics define which approach to take first?

2. What are patients' preferences in LTM vs. CIA as first-line therapies?

3. What is the optimal CIA regimen? Daily? Monthly cycles? Alternating CIA agents?

4. Are there predictors of CIA adverse events such a bronchospasm? Are CIA therapy test dose trials needed in all patients?

5. Do rapid delivery nebulisers confer an improved adherence rate? What is the role of intelligent nebulisers that collect compliance data in day-to-day practice?

6. Does CIA therapy have a role even in patients with known resistant strain to the agent (i.e. does suppressing the susceptible strains provide a benefit even if this selects out a resistant sub population)?

CIA: chronic inhaled antimicrobial; LTM: long-term macrolide.

Clinicians may well however prefer to limit LTM use and target pathogens with inhaled therapies. Shared decision making will ultimately improve patient adherence (Table 6).

\section{ADHERENCE AND EDUCATION}

For some patients with bronchiectasis, multiple medical treatments may be required. Some of these, such as nebulised treatments and airway clearance regimes, can be particularly burdensome in terms of a regular time commitment. It makes sense that for treatments to be effective, they need to be taken as prescribed, but what do we know about deviations from the prescribed regime and what effect this has in bronchiectasis? One recent study looked at treatment adherence in bronchiectasis and found that over a one-year period just over $50 \%$ of participants were adherent to inhaled antibiotics and other respiratory treatments and $41 \%$ adherent to airway 
clearance techniques. Less than $20 \%$ of the cohort were adherent to all treatments ${ }^{62}$. Adherence to inhaled antibiotics was associated with significantly fewer exacerbations.

A high degree of adherence seems to be beneficial, at least when it comes to inhaled antibiotics, so promoting treatment adherence and educating patients about this is important. The BTS guidelines recommend patient education about treatments and self-management, but no specific information or educational packages are recommended ${ }^{9}$. Qualitative studies highlight that patients feel that lack of information is one of the barriers to self-management and describe the importance of information in improving patients' confidence and in developing the skills to live with and manage their condition ${ }^{63,64}$. Factors predicting adherence to treatment in bronchiectasis include patients' beliefs about treatments, perceived treatment burden, and number of prescribed treatments ${ }^{65}$. Further work to develop a behaviour change intervention to promote adherence to treatment in bronchiectasis offers hope ${ }^{66}$.

Developing an understanding of patients' and their carer givers' information and education needs and how these could be addressed may offer a key advance ${ }^{67}$. A variety of packages have been developed for use in COPD, yet there remains to be an evidenced-based intervention designed for use in this patient population $^{68-70}$.

\section{CONCLUSIONS}

Patients with bronchiectasis and clinicians managing them are unable to achieve optimal patient care until major gaps in our understanding of the disease pathophysiology, prognosis, and optimal treatment regimens are advanced. Collaborative working and harnessing the enthusiasm and engagement of a patient population that has been frustrated by delays in diagnosis and empirical treatment into both observational and interventional studies will be crucial to achieving this. After the recently noted increases in bronchiectasis prevalence, the renewed enthusiasm from the life sciences industry for intervention studies in bronchiectasis and international collaborative efforts in bronchiectasis are both timely and desperately needed.

\section{DISCLOSURE OF INTEREST}

We acknowledge helpful discussions on the knowledge gaps in bronchiectasis with colleagues and collaborators within both bronchiectasis networks EMBARC (www.bronchiectasis.eu) and BronchUK (www.bronch.ac.uk). ADS acknowledges funding to the BronchUK network from the UK Medical Research Council (grant MR/L011263/1) and a prior Higher Education Funding Council (HFCE senior lectureship). KLMH acknowledges UK National Institute for Health Research (NIHR) Doctoral Research Fellowship funding. MJM acknowledges Doctoral training funding from the Health Research Board, Ireland and a European Respiratory Society/European Lung Foundation long-term training fellowship and consulting fees from Bayer pharmaceuticals.

\section{REFERENCES}

\footnotetext{
1. O'Leary CJ, Wilson CB, Hansell DM et al. Relationship between psychological well-being and lung health status in patients with bronchiectasis. Respir Med. 2002;96:686-92.

2. McDonnell MJ, Ward C, Lordan JL, Rutherford RM. Non-cystic fibrosis bronchiectasis. QJM. 2013;106:709-15.
} 
3. King P, Holdsworth S, Freezer N, Holmes P. Bronchiectasis. Intern Med J. 2006;36:729-37.

4. Hester KL, Macfarlane JG, Tedd H et al. Fatigue in bronchiectasis. QJM. 2012;105:235-40.

5. Cole PJ. Inflammation: a two-edged sword-the model of bronchiectasis. Eur J Respir Dis Suppl. 1986;147:6-15.

6. Chalmers JD, Hill AT. Mechanisms of immune dysfunction and bacterial persistence in non-cystic fibrosis bronchiectasis. Mol Immunol. 2013;55:27-34.

7. Shoemark A, Ozerovitch L, Wilson R. Aetiology in adult patients with bronchiectasis. Respir Med. 2007;101:1163-70.

8. Loebinger MR, Wells AU, Hansell DM et al. Mortality in bronchiectasis: a long-term study assessing the factors influencing survival. Eur Respir J. 2009;34:843-9.

9. Pasteur MC, Bilton D, Hill AT. British Thoracic Society guideline for non-CF bronchiectasis. Thorax. 2010;65(Suppl 1):i1-58.

10. Anwar GA, McDonnell MJ, Worthy SA et al. Phenotyping adults with non-cystic fibrosis bronchiectasis: a prospective observational cohort study. Respir Med. 2013;107:1001-7.

11. Li AM, Sonnappa S, Lex C et al. Non-CF bronchiectasis: does knowing the aetiology lead to changes in management? Eur Respir J. 2005;26:8-14.

12. Hill AT. Bronchiectasis: time to take action and implement the British Thoracic Society quality standards. Chron Respir Dis. 2012;9:211-12.

13. McDonnell MJ, Ahmed M, Das J et al. Hiatal hernias are correlated with increased severity of non-cystic fibrosis bronchiectasis. Respirology. 2015;20:749-57.

14. Otto H, Rein JG. Morphological definition and diagnosis of bronchiectasis. Beitr Pathol. 1971;143:70-83.

15. Roberts HR, Wells AU, Milne DG et al. Airflow obstruction in bronchiectasis: correlation between computed tomography features and pulmonary function tests. Thorax. 2000;55:198-204.

16. Gatheral T, Kumar N, Sansom B et al. COPD-related bronchiectasis; independent impact on disease course and outcomes. COPD. 2014;11:605-14.

17. Hurst JR, Elborn JS, De Soyza A; BRONCH-UK Consortium. COPD-bronchiectasis overlap syndrome. Eur Respir J. 2015;45:310-13.

18. Seitz AE, Olivier KN, Adjemian J, Holland SM, Prevots DR. Trends in bronchiectasis among medicare beneficiaries in the United States, 20002007. Chest. 2012;142:432-9.

19. Roberts ME, Lowndes L, Milne DG, Wong CA. Socioeconomic deprivation, readmissions, mortality and acute exacerbations of bronchiectasis. Intern Med J. 2012;42:e129-36.

20. Ringshausen FC, de Roux A, Diel R et al. Bronchiectasis in Germany: a population-based estimation of disease prevalence. Eur Respir J. 2015. [Epub ahead of print].

21. Quint JK, Millett ERC, Joshi M et al. Changes in the incidence, prevalence and mortality of bronchiectasis in the UK from 2004 to 2013: a population-based cohort study. Eur Respir J 2015. [Epub ahead of print].

22. RESPIRE 1 study design. Available at: https: / / www.clinicaltrials.gov/ct2/ show $/$ NCT01764841?term $=$ bronchiectasis + respire\&rank=1 [Accessed 10 October 2015]

23. ORBIT 3 study design. Available at: https://www.clinicaltrials.gov/ct2/ show $/$ NCT01515007?term $=$ bronchiectasis+orbit\&rank $=2$ [Accessed 10 October 2015].

24. Martínez-García MÁ, de Gracia J, Vendrell Relat M et al. Multidimensional approach to non-cystic fibrosis bronchiectasis: the FACED score. Eur Respir J. 2014;43:1357-67.

25. Chalmers JD, Goeminne P, Aliberti S et al. The bronchiectasis severity index. An international derivation and validation study. Am J Respir Crit Care Med. 2014;189:576-85.

26. McDonnell MJ, Kester K, De Soyza A. Bronchiectasis: Clinical features and management with a focus on inhaled antibiotics. Clin Pulm Med. 2014;21:251-61.

27. Chalmers JD, Aliberti S, Blasi F. State of the art review: management of bronchiectasis in adults. Eur Respir J. 2015. [Epub ahead of print].

28. King PT, Hutchinson P, Holmes PW et al. Assessing immune function in adult bronchiectasis. Clin Exp Immunol. 2006;144:440-6.
29. Ruchaud-Sparagnano MH, Gertig H, Hester KL et al. Effect of granulocyte-macrophage colony stimulating factor on neutrophil function in idiopathic bronchiectasis. Respirology. 2013;18:1230-5.

30. Masekala R. The immunology of bronchiectasis: complex machinery unravelled. Curr Allergy Clin Immunol. 2013;26.

31. Voynow JA, Young LR, Wang $Y$ et al. Neutrophil elastase increases MUC5AC mRNA and protein expression in respiratory epithelial cells. Am J Physiol. 1999;276:L835-43.

32. Voglis S, Quinn K, Tullis E et al. Human neutrophil peptides and phagocytic deficiency in bronchiectatic lungs. Am J Respir Crit Care Med. 2009;180:159-66.

33. Nicotra MB, Rivera M, Dale AM et al. Clinical, pathophysiologic, and microbiologic characterization of bronchiectasis in an aging cohort. Chest. 1995;108:955-61.

34. Pasteur MC, Helliwell SM, Houghton SJ et al. An investigation into causative factors in patients with bronchiectasis. Am J Respir Crit Care Med. 2000;162:1277-84.

35. Finch S, McDonnell MJ, Abo-Leyah H, Aliberti S, Chalmers JD. A comprehensive analysis of the impact of pseudomonas aeruginosa colonisation on prognosis in adult bronchiectasis. Ann Am Thorac Soc. 2015;12:1602-11.

36. Saiman L, Siegel JD, LiPuma JJ et al. Infection prevention and contro guideline for cystic fibrosis: 2013 Update. Infect Control Hosp Epidemiol. 2014;35(Suppl 1):S1-67.

37. Bryant JM, Grogono DM, Greaves D et al. Whole-genome sequencing to identify transmission of Mycobacterium abscessus between patients with cystic fibrosis: a retrospective cohort study. Lancet. 2013;381:1551-60.

38. Bryant JM, Harris SR, Parkhill J et al. Whole-genome sequencing to estab lish relapse or re-infection with Mycobacterium tuberculosis: a retrospective observational study. Lancet Respir Med. 2013;1:786-92.

39. De Soyza A, Perry A, Hall AJ et al. Molecular epidemiological analysi suggests cross-infection with Pseudomonas aeruginosa is rare in non-cystic fibrosis bronchiectasis. Eur Respir J. 2014;43:900-3.

40. Pujana I, Gallego L, Martín G et al. Epidemiological analysis of sequential Pseudomonas aeruginosa isolates from chronic bronchiectasis patients without cystic fibrosis. J Clin Microbiol. 1999;37:2071-3.

41. Valderrey AD, Pozuelo MJ, Jiménez PA et al. Chronic colonization by Pseudomonas aeruginosa of patients with obstructive lung diseases: cystic fibrosis, bronchiectasis, and chronic obstructive pulmonary disease. Diagn Microbiol Infect Dis. 2010;68:20-7.

42. McDonnell MJ, Jary HR, Perry A et al. Non cystic fibrosis bronchiectasis: a longitudinal retrospective observational cohort study of Pseudomonas persistence and resistance. Respir Med. 2015;109:716-26

43. Orriols R, Hernando R, Ferrer A, Terradas S, Montoro B. Eradication therapy against Pseudomonas aeruginosa in non-cystic fibrosis bronchiectasis. Respiration. 2015;90:299-305.

44. Rogers GB, van der Gast CJ, Serisier DJ. Predominant pathogen competition and core microbiota divergence in chronic airway infection. ISME 2014;9:217-25

45. Rogers GB, Bruce KD, Martin ML, Burr LD, Serisier DJ. The effect of longterm macrolide treatment on respiratory microbiota composition in non-cystic fibrosis bronchiectasis: an analysis from the randomised, double-blind, placebo-controlled BLESS trial. Lancet Respir Med. 2014;2:988-96.

46. Tunney MM, Einarsson GG, Wei L et al. Lung microbiota and bacteria abundance in patients with bronchiectasis when clinically stable and during exacerbation. Am J Respir Crit Care Med. 2013;187:1118-26.

47. Purcell P, Jary H, Perry A et al. Polymicrobial airway bacterial communities in adult bronchiectasis patients. BMC Microbiol. 2014;14:130.

48. Gao Y, Guan W, Xu G et al. The role of viral infection in pulmonary exacerbations of bronchiectasis in adults: A prospective study. Chest. 2015;147:1635-43.

49. Barker AF, O'Donnell AE, Flume P et al. Aztreonam for inhalation solution in patients with non-cystic fibrosis bronchiectasis (AIR-BX1 and AIR-BX2) two randomised double-blind, placebo-controlled phase 3 trials. Lancet Respir Med. 2014;2:738-49.

50. Bilton D, Tino G, Barker AF et al. Inhaled mannitol for non-cystic fibrosis bronchiectasis: a randomised, controlled trial. Thorax. 2014;69:1073-9. 
51. Serisier DJ, Martin ML, McGuckin MA et al. Effect of long-term, low-dose erythromycin on pulmonary exacerbations among patients with non-cystic fibrosis bronchiectasis: the BLESS randomized controlled trial. JAMA. 2013;309:1260-7.

52. Altenburg J, de Graaff CS, Stienstra Y et al. Effect of azithromycin maintenance treatment on infectious exacerbations among patients with non-cystic fibrosis bronchiectasis: the BAT randomized controlled trial. JAMA. 2013;309:1251-9.

53. Wong C, Jayaram L, Karalus $\mathrm{N}$ et al. Azithromycin for prevention of exacerbations in non-cystic fibrosis bronchiectasis (EMBRACE): a randomised, double-blind, placebo-controlled trial. Lancet. 2012;380:660-7.

54. Zhuo GY, He Q, Xiang-Lian L, Ya-Nan Y, Si-Te F. Prolonged treatment with macrolides in adult patients with non-cystic fibrosis bronchiectasis: meta-analysis of randomised controlled trials. Pulm Pharmacol Ther. 2014;29:80-8.

55. Wu Q, Shen W, Cheng H, Zhou X. Long-term macrolides for non-cystic fibrosis bronchiectasis: a systematic review and meta-analysis. Respirology. 2014;19:321-9.

56. Gao YH, Guan WJ, Xu G, Chen RC. Macrolide treatment in patients with bronchiectasis: more attention should be paid to the number of exacerbations. Pulm Pharmacol Ther. 2014;27:213-14.

57. Shi ZL, Peng H, Hu XW, Hu JG. Effectiveness and safety of macrolides in bronchiectasis patients: a meta-analysis and systematic review. Pulm Pharmacol Ther. 2014;28:171-8.

58. Murray MP, Govan JRW, Doherty CJ et al. A randomised controlled trial of nebulised gentamicin in non-cystic fibrosis bronchiectasis. Am J Respir Crit Care Med. 2011;183:491-9.

59. Haworth CS, Foweraker JE, Wilkinson P, Kenyon RF, Bilton D. Inhaled colistin in patients with bronchiectasis and chronic Pseudomonas aeruginosa infection. Am J Respir Crit Care Med. 2014;189:975-82.

60. Serisier DJ, Bilton D, De Soyza A et al. Inhaled, dual release liposomal ciprofloxacin in non-cystic fibrosis bronchiectasis (ORBIT-2): a randomized, double-blind, placebo-controlled trial. Thorax. 2013;68:812-17.
61. Wilson R, Welte T, Polverino E et al. Ciprofloxacin dry powder for inhalation in non-cystic fibrosis bronchiectasis: a phase II randomized study. Eur Respir J. 2013;41:1107-15

62. McCullough AR, Tunney MM, Quittner AL et al. Treatment adherence and health outcomes in patients with bronchiectasis. BMC Pulm Med. 2014;14:107.

63. Lavery KA, O'Neill B, Parker M, Elborn JS, Bradley JM. Expert patient self-management program versus usual care in bronchiectasis: a randomized controlled trial. Arch Phys Med Rehabil. 2011;92:1194-201.

64. Hester KL, De Soyza A, Rapley T. Information and education needs of patients with bronchiectasis: A qualitative investigation. Thorax. 2012;67: A141.

65. McCullough AR, Tunney MM, Elborn JS et al. Predictors of adherence to treatment in bronchiectasis. Respir Med. 2015;109:838-45.

66. McCullough AR, Ryan C, O'Neill B et al. Defining the content and delivery of an intervention to Change AdhereNce to treatment in BonchiEctasis (CAN-BE): a qualitative approach incorporating the Theoretical Domains Framework, behavioural change techniques and stakeholder expert panels. BMC Health Serv Res. 2015;15:342.

67. Hester KLM, Newton J, De Souza A, Rapley, T. Living your life with bronchiectasis: an exploration of patients and carers information needs. In The British Sociological Association Medical Sociology 47th Annual Conference. 2015. P106. ISBN: 9780904569452.

68. Apps LD, Mitchell KE, Harrison SL et al. The development and pilot testing of the self-management programme of activity, coping and education for chronic obstructive pulmonary disease (SPACE for COPD). Int J Chron Obstruct Pulmon Dis. 2013;8:317-27.

69. Mitchell K, Johnson-Warrington V, Apps LD et al. A self-management programme for COPD: a randomised controlled trial. Eur Respir J. 2014 44:1538-47.

70. Cosgrove C, MacMahon J, Bourbeau J et al. Facilitating education in pulmonary rehabilitation using the Living Well with COPD programme for pulmonary rehabilitation: a process evaluation. BMC Pulm Med. 2013;13:5. 